\title{
Decreased Basal Ganglia Volume in Cerebral Amyloid Angiopathy
}

\author{
Panagiotis Fotiadis, ${ }^{\mathrm{a}, \mathrm{b}}$ Marco Pasi, ${ }^{\mathrm{a}, \mathrm{c}}$ Andreas Charidimou, ${ }^{\mathrm{a}}$ Andrew D. Warren, ${ }^{\mathrm{a}}$ Kristin M. Schwab, ${ }^{\mathrm{a}}$ \\ Alzheimer's Disease Neuroimaging Initiative, ${ }^{*}$ Jonathan Rosand, ${ }^{a}$ Jeroen van der Grond, ${ }^{\mathrm{d}}$ \\ Mark A. van Buchem, ${ }^{\mathrm{d}}$ Anand Viswanathan, ${ }^{\mathrm{a}}$ M. Edip Gurol, ${ }^{\mathrm{a}}$ Steven M. Greenberg ${ }^{\mathrm{a}}$ \\ ${ }^{a}$ Department of Neurology, J.P. Kistler Stroke Research Center, Massachusetts General Hospital, Harvard Medical School, Boston, MA, USA \\ ${ }^{b}$ Department of Neuroscience, Perelman School of Medicine, University of Pennsylvania, Philadelphia, PA, USA \\ 'Stroke Unit, Department of Neurology, University of Lille, INSERM U1171, CHU Lille, Lille, France \\ ${ }^{\mathrm{d}}$ Department of Radiology, Leiden University Medical Center, Leiden, the Netherlands
}

Background and Purpose Cerebral amyloid angiopathy (CAA) is a common pathology of the leptomeningeal and cortical small vessels associated with hemorrhagic and non-hemorrhagic brain injury. Given previous evidence for CAA-related loss of cortical thickness and white matter volume, we hypothesized that CAA might also cause tissue loss in the basal ganglia.

Methods We compared basal ganglia volumes expressed as a percentage of total intracranial volume (pBGV) of non-demented patients with sporadic and hereditary CAA to age-matched healthy control (HC) and Alzheimer's disease (AD) cohorts.

Results Patients with sporadic CAA had lower pBGV $(n=80,1.16 \% \pm 0.14 \%)$ compared to HC $(\mathrm{n}=80,1.30 \% \pm 0.13 \%, P<0.0001)$ and $\mathrm{AD}$ patients $(\mathrm{n}=80,1.23 \% \pm 0.11 \%, P=0.001)$. Similarly, patients with hereditary CAA demonstrated lower pBGV $(n=25,1.26 \% \pm 0.17 \%)$ compared to their matched $\mathrm{HC}(\mathrm{n}=25,1.36 \% \pm 0.15 \%, P=0.036)$. Using a measurement of normalized basal ganglia width developed for analysis of clinical-grade magnetic resonance images, we found smaller basal ganglia width in patients with CAA-related lobar intracerebral hemorrhage $(\mathrm{ICH} ; n=93$, $12.35 \pm 1.47)$ compared to age-matched patients with hypertension-related deep ICH ( $n=93$, $13.46 \pm 1.51, P<0.0001)$ or $\mathrm{HC}(\mathrm{n}=93,15.45 \pm 1.22, P<0.0001)$. Within the sporadic CAA research cohort, decreased basal ganglia volume was independently correlated with greater cortical gray matter atrophy ( $r=0.45, P<0.0001)$, increased basal ganglia fractional anisotropy $(r=-0.36$, $P=0.001)$, and worse performance on language processing $(r=0.35, P=0.003)$, but not with cognitive tests of executive function or processing speed.

Conclusions These findings suggest an independent effect of CAA on basal ganglia tissue loss, indicating a novel mechanism for CAA-related brain injury and neurologic dysfunction.
Correspondence: Steven M. Greenberg Department of Neurology, J. P. Kistler Stroke Research Center, Massachusetts General Hospital, Harvard Medical

School, 175 Cambridge Street, Suite 300, Boston, MA 02114, USA

Tel: +1-617-726-8459

Fax: +1-617-726-5346

E-mail: sgreenberg@mgh.harvard.edu

Received: October 15, 2020

Revised: December 16, 2020

Accepted: January 6, 2021

*Part of the data used in preparation of this article was obtained from the Alzheimer's Disease Neuroimaging Initiative (ADNI) database (adni.loni. usc.edu). As such, the investigators within the ADNI contributed to the design and implementation of ADNI and/or provided data but did not participate in analysis or writing of this report. A complete listing of ADNI investigators can be found at the online supplemental file.

Keywords Cerebral amyloid angiopathy; Basal ganglia; Atrophy; Diffusion; Cognition 


\section{Introduction}

Although brain atrophy, defined as loss of brain volume unrelated to a defined macroscopic injury, ${ }_{1}^{1}$ is a prominent feature of neurodegenerative disease, it can also reflect cerebrovascular disease. Reduced overall brain volume ${ }^{2}$ as well as specific reductions in white matter volume (WMV) ${ }^{3}$ or cortical thickness ${ }^{4}$ have been observed in various small vessel diseases, often correlating with measures of cognitive impairment. Atrophy of the basal ganglia, however, has generally not been analyzed in the setting of cerebral small vessel disease.

Cerebrovascular $\beta$-amyloid deposition or cerebral amyloid angiopathy (CAA) is a common pathology of the leptomeningeal and cortical small vessels that has been linked to cortical hemorrhagic lesions as well as non-hemorrhagic cortical injury such as brain atrophy. Analysis of patients diagnosed with sporadic CAA by validated clinical criteria or with hereditary CAA by genetic testing has found thinning of the cerebral cortex in both groups. ${ }^{5}$ Despite its localization to leptomeningeal and cortical small vessels, CAA also appears to cause substantial non-hemorrhagic damage to subcortical structures, including increased white matter hyperintensities of presumed vascular origin, ${ }^{6}$ lower supratentorial WMVs, ${ }^{7}$ changes in white matter diffusion-tensor imaging (DTI) properties, ${ }^{8,9}$ and white matter microinfarcts. ${ }^{10,11}$ The likeliest mechanism for CAA-related non-hemorrhagic brain injury appears to be vascular dysfunction ${ }^{12,13}$ leading to tissue ischemia. Although few studies have searched for effects of CAA on subcortical gray matter structures such as the basal ganglia, an early DTI study of this disorder reported increased fractional anisotropy (FA) of subthalamic gray matter. ${ }^{8}$

Based on the association of CAA with volume loss in cortex and white matter, we sought to determine its relationship to basal ganglia volume (BGV). The basal ganglia, typically defined as putamen, caudate, globus pallidus, and nucleus accumbens, are a set of interconnected deep gray matter nuclei thought to regulate a range of complex functions including voluntary and involuntary motor control, ${ }^{14}$ non-declarative memory and habit formation, ${ }_{1}^{15}$ language processing ${ }_{1}^{16}$ action selection, ${ }^{17}$ and emotional perception. ${ }^{18}$ Basal ganglia damage is a prominent early manifestation of neurodegenerative disorders such as Parkinson's disease, ${ }_{1}^{19}$ progressive supranuclear palsy, ${ }_{1}^{20}$ and Huntington's disease, ${ }^{21}$ where it is felt to mediate neurocognitive as well as movement-related clinical symptoms. ${ }^{22}$ From a clinical standpoint, the current finding of CAA-related loss of BGV could represent a novel mechanism for mediating a portion of the cognitive and motor impairments related to this common small vessel pathology and thus an imaging marker for assessing CAA's impact and its response to candidate interventions.

\section{Methods}

\section{Study design and participants}

Research cohorts

Individuals ( $n=80$ ) with sporadic CAA were prospectively enrolled at Massachusetts General Hospital, in Boston, MA, between March 15, 2006 and December 1, 2014. Each individual was non-demented and carried a diagnosis of probable CAA per the pathologically validated modified Boston criteria. ${ }^{23}$ Demographic and clinical data were collected for each patient at the time of enrollment.

Of the 80 research patients with sporadic CAA, 68 agreed to undergo a standard neurocognitive battery. Executive function (Trail Making test B, Digit Span test backwards, verbal fluency test), processing speed (Trail Making Test A, Symbol Substitution test), verbal memory (immediate and delayed memory scores based on Hopkins Verbal Learning test), and language processing (Animal Naming test, Boston naming test) z-scores were calculated using the mean and standard deviation scores from the entire sporadic CAA cohort, as described. ${ }^{9}$ Gait velocity and time were determined using the Timed Get Up and Go test $(n=62){ }^{9}$

Individuals in the research CAA cohort were age-matched to healthy controls $(\mathrm{HC} ; \mathrm{n}=80)$ and patients with Alzheimer's disease ( $A D ; n=80$ ) collected from the Alzheimer's Disease Neuroimaging Initiative (ADNI). ${ }^{24}$ Of the 80 AD patients collected from $A D N I, 46$ (57.5\%) had a clinical dementia rating score of 0.5 ; the remaining 34 had a clinical dementia rating score of 1 .

Individuals with the Dutch-type hereditary form of CAA (DCAA, previously known as Hereditary Cerebral Hemorrhage With Amyloidosis-Dutch or HCHWA-D) were prospectively enrolled at the Leiden University Medical Center and the diagnosis confirmed by genetic testing. ${ }^{5,25}$ The D-CAA patients $(n=25)$ were age-matched to genetically verified mutationnegative HCs $(n=25)$ enrolled at the same center. Of the 25 enrolled D-CAA patients, 14 presented clinically with lobar intracerebral hemorrhage $(\mathrm{ICH})$ and 10 were presymptomatic carriers of the D-CAA-associated gene mutation. Demographic and clinical data were similarly collected for the D-CAA patients. ${ }^{25}$

Cohorts with clinical grade magnetic resonance images In addition to the above cohorts that received high-resolution 
dedicated research magnetic resonance images (MRIs), we analyzed clinical MRIs of patients who were admitted to Massachusetts General Hospital between January, 2003 and February, 2012, with spontaneous symptomatic ICH. Patients in this group were categorized as having definite or probable CAA-related lobar ICH (CAA-ICH: $n=93$ ), and age-matched patients with hypertensive arteriopathy and ICH (HTN-ICH: $n=93)$. The latter group consisted of patients with ICH in the basal ganglia, thalamus, or brainstem but not lobar $\mathrm{ICH}$, as described. ${ }^{26}$ All analyses were performed on non-ICH containing hemispheres; patients with bilateral ICHs were excluded. Demographic and clinical information was obtained for those patients at the time of enrollment. ${ }^{26}$

Age-matching was performed based on the closest first decimal of age without knowledge of clinical or radiological information. ${ }^{5}$ The Institutional Review Boards of the participating sites approved this study and all patients provided signed written informed consent.

\section{MRI acquisition}

The research cohort of sporadic CAA patients underwent structural MRI using a Siemens Avanto 1.5T scanner (12-channel head coil; Siemens Healthineers, Erlangen, Germany). The standardized battery included T1-weighted Multi-echo Magnetization-Prepared Rapid Gradient Echo (voxel size $1 \times 1 \times 1 \mathrm{~mm}^{3}$; repetition time [TR], 2,730 ms; inversion time [TI], 1,100 ms), high-resolution susceptibility weighted imaging ( $\mathrm{SWl}$; voxel size $0.75 \times 0.75 \times 1.3 \mathrm{~mm}^{3}$; TR, $48 \mathrm{~ms}$; echo time [TE], $40 \mathrm{~ms}$ ), three-dimensional fluid attenuated inversion recovery (FLAIR; voxel size $1 \times 1 \times 1 \mathrm{~mm}^{3}$; TR, 6,000 ms; TE, $302 \mathrm{~ms}$; TI, 2,200 ms), and high-resolution Diffusion (voxel size $2 \times 2 \times 2 \mathrm{~mm}^{3}$; TR, 8,180 ms; TE, $82 \mathrm{~ms} ; 60$ directions; b-value $=700 ; 10$ bo images) sequences.

$H C s$ and patients with $A D$ selected from the ADNI database underwent a similar structural MRI protocol using Siemens 1.5T scanners. ${ }^{5,24}$

Patients diagnosed with D-CAA and their matched controls underwent research structural MRI using a Philips $3 T$ scanner (Philips Healthcare, Andover, MA, USA). The MRI protocol included 3D T1-weighted (voxel size $0.875 \times 0.875 \times 1.2 \mathrm{~mm}^{3}$; TR, $9 \mathrm{~ms} ; \mathrm{TE}, 4.6 \mathrm{~ms}$ ), SWI (voxel size $0.7812 \times 0.7812 \times 0.8 \mathrm{~mm}^{3}$; TR, $45 \mathrm{~ms} ; \mathrm{TE}, 31 \mathrm{~ms}$ ), and FLAIR (voxel size $0.4297 \times 0.4297 \times 5.5$ $\mathrm{mm}^{3}$; TR, $11 \mathrm{~ms} ; \mathrm{TE}, 125 \mathrm{~ms}$ ) sequences.

The clinical cohorts of patients with CAA-ICH or HTN-ICH underwent a structural MRI protocol using a General Electric Sigma 1.5T scanner (GE Healthcare, Barrington, IL, USA). The clinical MRI protocols included T1-weighted, T2*-weighted Gradient-Recalled Echo, and FLAIR sequences, as described. ${ }^{26}$

\section{MRI processing}

\section{Structural analyses}

Segmentation of cortical gray, white, and basal ganglia structures as well as estimated total intracranial volume (eTIV) were performed using FreeSurfer version 5.3.0 (https://surfer.nmr. mgh.harvard.edu). Cortical gray matter volume (CGMV) was defined as the volume between the pial surface and gray-white junction, measured using a surface-based approach ${ }^{27}$ and expressed as a percent of intracranial volume (pCGMV). Similarly, WMV was defined as the volume enclosed within the graywhite junction minus any non-white matter entities such as the subcortical gray matter structures and the ventricles and expressed as a percent of intracranial volume (pWMV). The basal ganglia volume was defined as the sum of the putamen, caudate nucleus, globus pallidus, and nucleus accumbens volumes, and expressed as a percent of intracranial volume (pBGV). White matter hyperintensity volume, a marker of CAA-related structural injury, was estimated using a previously validated semi-automated algorithm, and expressed as a percentage of eTIV (pWMH). ${ }^{6}$

\section{Diffusion analyses}

High-resolution diffusion scans were preprocessed using the 'dt_recon' pipeline of FreeSurfer. Preprocessing of the scans consisted of correcting eddy currents and head motion artifacts while registering all diffusion scans to a common anatomical template. After manually confirming accurate co-registration between the diffusion and anatomical scans, the resulting FA and apparent diffusion coefficient (ADC) volumetric heat maps were used to calculate the FA and ADC values within the putamen, caudate nucleus, globus pallidus, and nucleus accumbens. Basal ganglia FA and ADC values were defined as the volume-weighted average $F A$ and $A D C$ of those sub-structures.

All segmentation outputs and results were manually checked to ensure accuracy and manual interventions were performed when necessary. ${ }^{5}$ Whenever gross pathology (i.e., $\mathrm{ICH}$ ) was present in either hemisphere $(n=40)$, results were obtained only from the contralateral hemisphere.

\section{Normalized basal ganglia width}

To assess basal ganglia atrophy in ICH patients with clinical-grade MRI scans not suitable for FreeSurfer-based volumetric measurement, we developed a metric based on the sum of the widths of three basal ganglia substructures-the caudate nucleus, putamen, and globus pallidus-normalized to the intracranial diameter (Figure 1). The measurement was obtained 
from the axial slice containing the largest area of the putamen, anterior commissure, septum pellucidum, and the pulvinar of thalamus ${ }^{28}$ in the ICH-free hemisphere of each subject's axial T1-weighted sequence. An experienced rater (P.F.) determined normalized basal ganglia width on the $93 \mathrm{CAA}-\mathrm{ICH}$ and 93 HTN-ICH clinical cases of interest. For comparison to HCs, we applied the same basal ganglia width metric to MRI scans from $93 \mathrm{HC}$ obtained from ADNI (Table 1). To test the metric's reliability, a second rater (M.P.) independently measured 30 randomly selected CAA-ICH cases and 30 randomly selected HTN$\mathrm{ICH}$ cases. For these 60 scans, the intraclass correlation coefficient (two-way random effects model, consistency, single measures; SPSS software suite version 25, IBM Co., Armonk, NY, USA) was 0.847 (95\% confidence interval [Cl], 0.756 to
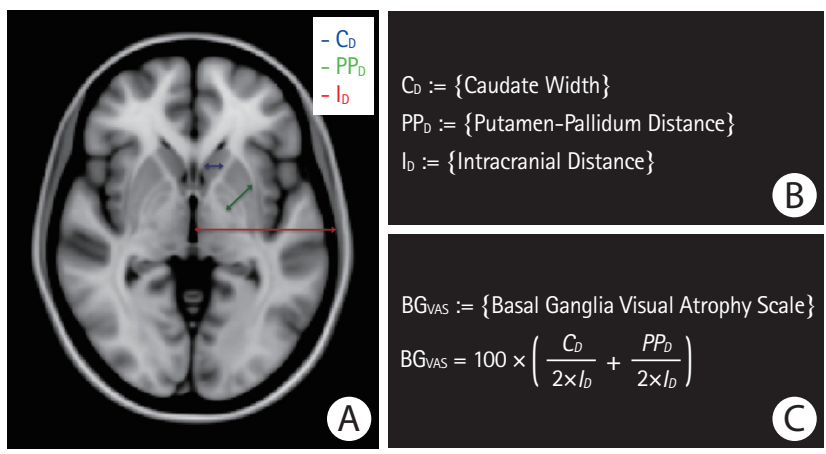

Figure 1. Visual basal ganglia atrophy scale. (A) Paradigm illustrating the three widths of interest: estimated caudate width (indicated in blue), estimated putamen-pallidum distance (indicated in green), and estimated hemispheric intracranial distance (indicated in red). (B) Definitions of the three widths of interest. (C) Definition of the normalized basal ganglia visual atrophy scale.
0.906). To assess the validity of the developed basal ganglia visual atrophy scale to accurately estimate BGV, we applied it on the research-quality ADNI HC cohort $(n=93)$ and compared it to their corresponding FreeSurfer-derived pBGV. The Pearson's correlation between the visual atrophy scale and the automated volumetric measurements was $r=0.22(P=0.037)$.

\section{Statistical analyses}

Statistical analyses were performed using the JMP Statistical Software by SAS version Pro 14.0 (SAS Institute Inc., Cary, NC, USA). Bivariate correlation analyses were carried out in the form of two-tailed t-tests (threshold for significance, $P<0.05$ ), and Pearson's correlation coefficients $(r)$. The 240 research subjects (80 sporadic CAA patients, 80 matched HC, and 80 matched $A D$ patients) were included in a general linear model with $\mathrm{pBGV}$ as the dependent variable, the binary diagnostic status ("CAA" vs. "HC" vs. "AD") as the class variable, and other potential confounders (age, sex, presence of hypertension, hyperlipidemia, and diabetes mellitus) as covariates. Tukey's test was used to correct for multiple comparisons between the three groups. Multiple linear regression models were additionally implemented to examine the independent relationship between the dependent variable $\mathrm{pBGV}$ and other variables of interest, after adjusting for the potentially confounding effects of age, sex, presence of hypertension, hyperlipidemia, and diabetes mellitus. Standardized regression coefficients ( $\beta$ ), 95\% $\mathrm{Cl}$, and $P$-values are reported for each multiple regression model. All volumetric variables examined were normally distributed.

A separate Bayesian appearance model was implemented

Table 1. Comparison in normalized basal ganglia width in clinical cohorts

\begin{tabular}{lccc}
\hline Variable & CAA-ICH & HTN-ICH & Healthy controls \\
\hline No. of individuals & 93 & 93 & 93 \\
Demographics & & & $73.1 \pm 3.3$ \\
Age (yr) & $68.5 \pm 7.1^{*}$ & $68.5 \pm 12.3^{*}$ & $43(46.2)$ \\
Female sex & $44(47.3)$ & $43(46.2)$ & $0(0)$ \\
Hypertension & $61(65.6)^{+, .+}$ & $75(80.7)^{+}$ & $42(45.2)$ \\
Hyperlipidemia & $40(43)$ & $38(40.9)$ & $9(9.7)$ \\
Diabetes mellitus & $11(11.8)^{+}$ & $24(25.8)^{+}$ & $0(0)$ \\
Previous hemorrhagic stroke & $17(19.5)^{+, \S}$ & $8(9.1)^{+}$ & $0(0)$ \\
Previous ischemic stroke & $6(6.5)^{+}$ & $10(10.8)^{+}$ & $15.45 \pm 1.22$ \\
Basal ganglia visual atrophy scale & & & $13.46 \pm 1.51^{*}$ \\
$\quad$ Normalized basal ganglia width & $12.35 \pm 1.47^{*,+}$ & & \\
\hline
\end{tabular}

Values are presented as mean \pm standard deviation or number (\%).

$\mathrm{CAA}-\mathrm{ICH}$, cerebral amyloid angiopathy-related intracerebral hemorrhage; $\mathrm{HTN}-\mathrm{ICH}$, hypertensive arteriopathy-related intracerebral hemorrhage.

${ }^{*}$ Denotes significantly less than healthy controls $(P \leq 0.002) ;{ }^{\dagger}$ Denotes significantly higher than Healthy Controls $(P<0.05) ;{ }^{*}$ Denotes significantly less than patients with HTN-ICH $(P \leq 0.002) ;{ }^{\S}$ Denotes significantly higher than patients with $\mathrm{HTN}-\mathrm{ICH}(P<0.05)$. 
using Functional Magnetic Resonance Imaging of the Brain (FMRIB) Software Library (FSL) FMRIB's Integrated Registration and Segmentation Tool (FIRST) automated toolbox in order to schematically explore the regional differences in volume between the basal ganglia and their individual components in patients with $C A A, H C$, and patients with $A D$. Topographical maps were generated using a threshold of $P<0.05$, corrected for multiple comparisons.

\section{Results}

\section{Decreased basal ganglia volume in CAA}

The research cohort consisted of 80 non-demented patients with sporadic CAA (mean age, $70.3 \pm 8.5$ years), 80 agematched $\mathrm{HC}(71.8 \pm 3.7)$, and 80 age-matched patients with $A D$ (70.4 \pm 6.8 ). Detailed demographic and clinical information for these cohorts are provided in Table 2.

Sporadic CAA patients had lower pBGV than both HC (10.7\% lower, $P<0.0001)$ and patients with $\operatorname{AD}(5.4 \%$ lower, $P=0.001)$. Atrophy was identified in the putamen, caudate nucleus, and globus pallidus (Table 3, Figures 2 and 3). Reduced pBGV in CAA relative to $\mathrm{HC}$ (pairwise difference $=-0.13 ; 95 \% \mathrm{Cl}_{1}-0.18$ to $-0.09 ; P<0.0001$ ) and $A D$ (pairwise difference $=-0.05 ; 95 \%$ $\mathrm{Cl}_{1}-0.10$ to $\left.-0.01 ; P=0.016\right)$ remained independent after controlling for age, sex, presence of hypertension, hyperlipidemia, and diabetes mellitus, using a general linear model adjusting for multiple comparisons (Tukey's). Other variables in this model correlating with decreased pBGV were male sex (pairwise difference $=-0.10 ; 95 \% \mathrm{Cl}_{1}-0.13$ to $-0.07 ; P<0.0001$ ) and presence of hypertension (pairwise difference $=-0.05 ; 95 \% \mathrm{Cl}$, -0.08 to $-0.01 ; P=0.005)$.

In a secondary analysis of basal ganglia atrophy relative to total brain atrophy, BGV was expressed as a percentage of total brain volume (defined as the sum of cortical gray matter and WMVs) and compared between the three cohorts. In bivariate analyses, patients with CAA had lower BGV expressed as a per- centage of total brain volume compared to both $\mathrm{HC}(4.1 \%$ lower, $P=0.015)$ and patients with $A D(3.8 \%$ lower, $P=0.024)$. The association between BGV expressed as a percentage of total brain volume and diagnostic group did not remain significant at the $P<0.05$ threshold (CAA vs. HC pairwise difference $=-0.08 ; 95 \% \mathrm{Cl},-0.17$ to $0.01 ; P=0.08$ ) (CAA vs. AD pairwise difference $=-0.07 ; 95 \% \mathrm{Cl}_{1}-0.15$ to $0.02 ; P=0.17$ ) after correcting for age, sex, presence of hypertension, hyperlipidemia, and diabetes mellitus (general linear model adjusting for multiple comparisons - Tukey's).

To further exclude confounding due to the presence of $\mathrm{ICH}$ or hypertension, we repeated these analyses in the subgroup of 40 CAA patients (mean age, $71.3 \pm 8.1$ ) without ICH versus their corresponding age-matched $\mathrm{HC}$ and $\mathrm{AD}$ patients, and the subgroup of 34 non-hypertensive patients with sporadic CAA $(67.9 \pm 8.3)$ versus their corresponding non-hypertensive agematched $H C$ and $A D$ patients. In both subgroup analyses, patients with sporadic CAA had lower pBGV versus their comparator subgroups (Table 3).

We performed similar analyses of BGV in patients with $D-C A A(n=25$; mean age, 46.0 \pm 14.2 ) and their corresponding age-matched HC $(n=25$; mean age, $46.0 \pm 11.5)$. D-CAA patients demonstrated lower $\mathrm{pBGV}$ than their matched $\mathrm{HC}$ (decrease by $7.2 \%, P=0.036$ ) (Table 3 ). This association was again independent of age, sex, presence of hypertension, hyperlipidemia, and diabetes mellitus (pairwise difference $=-0.09 ; 95 \% \mathrm{Cl},-0.17$ to $-0.01 ; P=0.029$ ). In the same model, increased age was also independently correlated with smaller pBGV $(\beta=-0.43 ; 95 \% \mathrm{Cl}$, -0.62 to $-0.14 ; P=0.003$ ). Decreased $p B G V$ in the $D-C A A$ individuals was localized to the putamen $(P=0.030)$ and the nucleus accumbens $(P=0.049)$ with trend towards smaller globus pallidus ( $P=0.083$ ) but not caudate nucleus ( $P=0.42$ ).

To analyze for basal ganglia atrophy using clinical (rather than research-quality) MRI scans from patients with CAA-ICH and HTN-ICH, we developed a reproducible technique (see Methods) for measuring the normalized width of the caudate,

Table 2. Demographics and clinical information in research cohorts

\begin{tabular}{|c|c|c|c|c|c|}
\hline \multirow{2}{*}{ Variable } & \multicolumn{3}{|c|}{ Sporadic cohorts } & \multicolumn{2}{|c|}{ Dutch cohorts } \\
\hline & Sporadic CAA & Healthy controls & $A D$ & D-CAA & Healthy controls \\
\hline No. of individuals & 80 & 80 & 80 & 25 & 25 \\
\hline Age (yr) & $70.3 \pm 8.5$ & $71.8 \pm 3.7$ & $70.4 \pm 6.8$ & $46 \pm 14.2$ & $46 \pm 11.5$ \\
\hline Female sex & $18(22.5)$ & $36(45.0)$ & 35 (43.8) & $15(60.0)$ & $17(68.0)$ \\
\hline Hypertension & $46(57.5)$ & $30(37.5)$ & 39 (48.8) & $6(24.0)$ & $4(16.0)$ \\
\hline Hyperlipidemia & $36(45.0)$ & $42(52.5)$ & $45(57.0)$ & $5(20.0)$ & $2(8.0)$ \\
\hline Diabetes mellitus & $6(7.5)$ & $9(11.3)$ & $6(7.6)$ & $2(8.0)$ & $0(0)$ \\
\hline
\end{tabular}

Values are presented as mean \pm standard deviation or number (\%).

CAA, cerebral amyloid angiopathy; AD, Alzheimer's disease; D-CAA, Dutch-type hereditary cerebral amyloid angiopathy. 
Table 3. Basal ganglia volumes in research cohorts

\begin{tabular}{|c|c|c|c|c|c|}
\hline \multirow{2}{*}{ Variable } & \multicolumn{3}{|c|}{ Sporadic cohorts } & \multicolumn{2}{|c|}{ Dutch cohorts } \\
\hline & Sporadic CAA & Healthy controls & $A D$ & D-CAA & Healthy controls \\
\hline \multicolumn{6}{|l|}{ All individuals } \\
\hline Basal ganglia volume (\% of eTIV) & $1.16 \pm 0.14^{*, t}$ & $1.30 \pm 0.13$ & $1.23 \pm 0.11^{*}$ & $1.26 \pm 0.17^{*}$ & $1.36 \pm 0.15$ \\
\hline Putamen volume (\% of eTIV) & $0.54 \pm 0.08^{*,+}$ & $0.60 \pm 0.07$ & $0.57 \pm 0.07^{*}$ & $0.58 \pm 0.12^{*}$ & $0.64 \pm 0.09$ \\
\hline Caudate volume ( $\%$ of eTIV) & $0.40 \pm 0.07^{*,+}$ & $0.46 \pm 0.06$ & $0.43 \pm 0.05^{*}$ & $0.46 \pm 0.05$ & $0.48 \pm 0.06$ \\
\hline Pallidus volume (\% of eTIV) & $0.17 \pm 0.02^{*,+}$ & $0.19 \pm 0.02$ & $0.18 \pm 0.02$ & $0.16 \pm 0.03$ & $0.17 \pm 0.02$ \\
\hline Accumbens volume ( $\%$ of eTIV) & $0.053 \pm 0.015$ & $0.056 \pm 0.012$ & $0.047 \pm 0.011^{*, *}$ & $0.055 \pm 0.015^{*}$ & $0.063 \pm 0.013$ \\
\hline \multicolumn{6}{|l|}{$\begin{array}{l}\text { Sporadic CAA without intracerebral } \\
\text { hemorrhage ( } n=40 \text { for each group) }\end{array}$} \\
\hline Basal ganglia volume (\% of eTIV) & $1.16 \pm 0.15^{*,+}$ & $1.30 \pm 0.14$ & $1.22 \pm 0.09^{*}$ & - & - \\
\hline \multicolumn{6}{|l|}{$\begin{array}{l}\text { Sporadic CAA without hypertension } \\
\text { ( } n=34 \text { for each group) }\end{array}$} \\
\hline Basal ganglia volume (\% of eTIV) & $1.16 \pm 0.16^{*, t}$ & $1.28 \pm 0.13$ & $1.24 \pm 0.12$ & - & - \\
\hline
\end{tabular}

Values are presented as mean \pm standard deviation.

CAA, cerebral amyloid angiopathy; AD, Alzheimer's disease; D-CAA, Dutch-type hereditary cerebral amyloid angiopathy; eTIV, estimated total intracranial volume. ${ }^{*}$ Denotes significantly less than healthy controls $(P<0.05) ;{ }^{~}$ Denotes significantly less than patients with $A D(P<0.05) ;{ }^{*}$ Denotes significantly less than patients with CAA $(P<0.05)$.

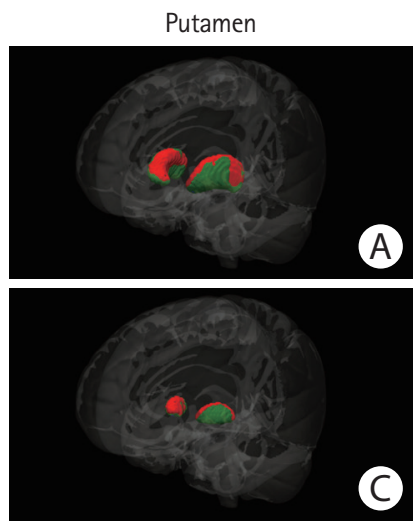

Globus pallidus

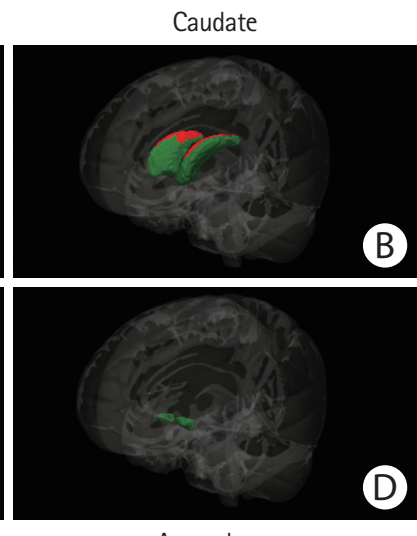

Accumbens

Figure 2. Regional basal ganglia atrophy in patients with sporadic cerebral amyloid angiopathy (CAA) versus healthy controls. The colored (green and red) regions represent masks of the (A) putamen, (B) caudate nucleus, (C) globus pallidus, or (D) nucleus accumbens. Red regions represent the locations where patients with sporadic CAA demonstrated significant atrophy when compared to age-matched healthy controls $(P<0.05$, corrected for multiple comparisons). Green regions represent the locations with no significant difference between the compared groups. The Functional Magnetic Resonance Imaging of the Brain (FMRIB) Software Library (FSL) FMRIB's Integrated Registration and Segmentation Tool (FIRST) for automatic segmentation of subcortical structures was used to generate the results. All results were manually checked to ensure accuracy, and the statistical maps were corrected for total intracranial volume.

putamen, and globus pallidus. Patients with CAA-ICH ( $n=93$; mean age, $68.5 \pm 7.1)$ were compared to similar aged patients with HTN-ICH $(n=93 ; 68.5 \pm 12.3)$ and an ADNI HC cohort $(n=93 ; 73.1 \pm 3.3)$ (Table 1). Patients with CAA-ICH had greater

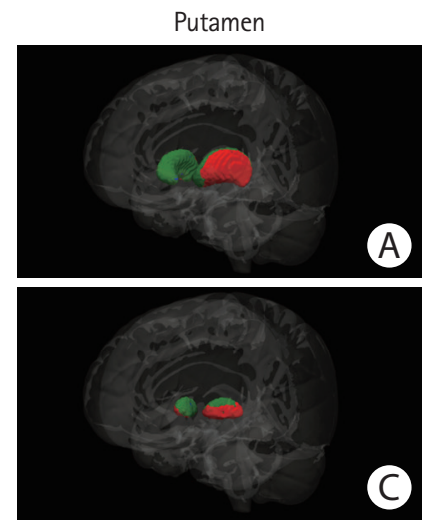

Globus pallidus

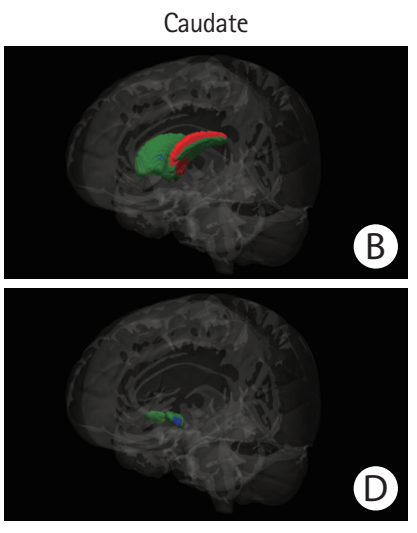

Accumbens
Figure 3. Regional basal ganglia atrophy in patients with sporadic cerebral amyloid angiopathy (CAA) versus patients with Alzheimer's disease (AD). The colored (green, red, and blue) regions represent masks of the (A) putamen, (B) caudate nucleus, (C) globus pallidus, or (D) nucleus accumbens. Red regions represent the locations where patients with sporadic CAA demonstrated significant atrophy when compared to age-matched patients with $A D$, blue regions the locations where patients with $A D$ demonstrated significant atrophy when compared to their matched CAA patients $(P<0.05$, corrected for multiple comparisons). Green regions represent the locations with no significant difference between the compared groups. The Functional Magnetic Resonance Imaging of the Brain (FMRIB) Software Library (FSL) FMRIB's Integrated Registration and Segmentation Tool (FIRST) for automatic segmentation of subcortical structures was used to generate the results. All results were manually checked to ensure accuracy, and the statistical maps were corrected for total intracranial volume.

basal ganglia atrophy versus HTN-ICH (8.3\% decrease in normalized basal ganglia width, $P<0.0001)$ and both $\mathrm{ICH}$ groups had greater basal ganglia atrophy versus HC (CAA-ICH vs. HC: 20.1\% decrease, HTN-ICH vs. HC: $12.9 \%$ decrease, $P<0.0001$ 
for both comparisons) (Table 1). Using a general linear model adjusting for multiple comparisons, the association between CAA-ICH and greater basal ganglia atrophy was again independent after correcting for age, sex, presence of hypertension, hyperlipidemia, and diabetes mellitus, as well as history of prior $\mathrm{ICH}$ and ischemic stroke (CAA-ICH vs. HTN-ICH pairwise difference $=-1.11 ; 95 \% \mathrm{Cl},-1.61$ to -0.62 and $\mathrm{CAA}-\mathrm{ICH}$ vs. $\mathrm{HC}$ pairwise difference $=-3.18 ; 95 \% \mathrm{Cl},-3.82$ to $-2.53 ; P<0.0001$ for both comparisons). In the same model, male sex was independently associated with decreased BGV (pairwise difference $=0.84 ; 95 \% \mathrm{Cl}, 0.52$ to $1.17 ; P<0.0001)$.

\section{Basal ganglia volume and other markers of structural integrity}

We previously reported CAA-related cortical and white matter atrophy $y^{5,7}$ as well as elevations in subcortical gray matter $F^{8}$ and explored the association of these parameters with CAA-related basal ganglia atrophy. Among sporadic CAA patients, reduced pBGV correlated with pCGMV $(r=0.45, P<0.0001)$ but not pWMV $(r=0.18, P=0.12)$ or $p W M H(r=0.02, P=0.85)$. The positive correlation between $\mathrm{pBGV}$ and $\mathrm{pCGMV}$ remained independent after adjusting for age, sex, presence of hypertension, hyperlipidemia, and diabetes mellitus ( $\beta=0.33 ; 95 \% \mathrm{Cl}, 0.05$ to $0.65 ; P=0.011$ ). The same subjects demonstrated a negative correlation between $\mathrm{pBGV}$ and basal ganglia FA ( $r=-0.36$, $P=0.001)$, and no association between $\mathrm{pBGV}$ and basal ganglia $A D C(r=0.05, P=0.68)$. The correlation between $p B G V$ and basal ganglia FA was also independent of age, sex, presence of hypertension, hyperlipidemia, and diabetes mellitus $(\beta=-0.24$; $95 \% \mathrm{Cl},-0.45$ to $0.03 ; P=0.031$ ).

\section{Basal ganglia volume and neurologic function}

Because of the association of CAA with cognitive impairment, ${ }_{1}^{29}$ we performed an exploratory analysis of the relationship between BGV and cognitive performance. Among the research cohort sporadic CAA patients who underwent complete neurocognitive assessment $(n=68)$, there was a positive correlation between $\mathrm{pBGV}$ and verbal memory $(\mathrm{r}=0.26, P=0.034)$ and language processing $(r=0.35, P=0.003)$, but not with executive function $(r=-0.05, P=0.67)$ or processing speed $(r=-0.13$, $P=0.30$ ). The positive association between $\mathrm{pBGV}$ and language processing was independent of age, sex, presence of hypertension, hyperlipidemia, diabetes mellitus, years of education, as well as pCGMV and pWMV $(\beta=0.35 ; 95 \% \mathrm{Cl}, 0.04$ to 0.66 ; $P=0.013$ ) whereas the association with verbal memory did not persist in this model $\left(\beta=0.13 ; 95 \% \mathrm{Cl}_{1}-0.23\right.$ to $\left.0.44 ; P=0.44\right)$. There was no association between $\mathrm{pBGV}$ and gait velocity $(r=0.02, P=0.91)$ or gait time $(r=-0.13, P=0.30)$.

\section{Discussion}

Our primary finding is that CAA is associated with reduced BGV. Total BGV was reduced by $10.7 \%$ and $7.2 \%$ respectively in non-demented sporadic and hereditary CAA patients relative to age-matched $\mathrm{HCs}$, with similar decreases observed in the individual volumes of putamen, caudate, and globus pallidus. BGV as a whole and the individual putamen, caudate, and globus pallidus volumes were also reduced in $C A A$ relative to $A D$ patients, while nucleus accumbens was smaller in $A D$ than $C A A$, consistent with the high burden of $A D$ pathology in this structure. ${ }^{30}$ Measuring the normalized widths of caudate, putamen, and globus pallidus, we found basal ganglia atrophy in both CAA-ICH and $\mathrm{HTN}-\mathrm{ICH}$ relative to $\mathrm{HCS}$, but to a greater extent in $\mathrm{CAA}-\mathrm{ICH}$ than $\mathrm{HTN}-\mathrm{ICH}$.

Effects of CAA on BGV are somewhat unexpected given CAA's primarily cortical and leptomeningeal localization; the current findings should therefore be considered exploratory and requiring of replication. CAA-related effects on subcortical brain regions such as the white matter, however, are well established. Amyloid positron emission tomography imaging, for example, showed a direct relationship between increasing brain amyloid burden and volume of white matter hyperintensity in non-demented CAA patients but not AD patients. ${ }^{6}$ The mechanisms for these distant effects on subcortical brain regions remain undetermined, but could include (1) subcortical ischemia due to cortical vascular dysfunction and distal hypoperfusion and (2) subcortical neurodegeneration due to loss of cortical afferent and efferent connections. Indeed, our primary and secondary analyses together indicate that basal ganglia atrophy in CAA is to some extent in proportion to total brain volume loss, which might result either from loss of afferent and efferent connections across compartments or from correlations in the extent of injury among the brain compartments driven by overall CAA severity in each brain. An early postmortem study of eight brains with CAA noted demyelination, vacuolation, and lacunae in white matter and basal ganglia in a pattern similar to Binswanger's subcortical encephalopathy, ${ }_{1}^{31}$ while another postmortem study of 18 brains with severe CAA found frequent microinfarcts in the white matter as well as cortical gray matter. ${ }^{10}$ Moreover, CAA-ICH patients have previously been reported to have a similar burden of WMH as HTN-ICH ${ }^{32}$ and in the current study demonstrate greater basal ganglia atrophy. These findings suggest that the relatively distant effects of CAA on subcortical brain structures equal or exceed those caused by arteriolosclerosis and lipohyalinosis of the more proximate deep penetrating arterioles in HTN-ICH. ${ }^{33}$ 
We found that reduced BGV among the sporadic CAA research cohort correlated with higher basal ganglia FA. DTIbased metrics such as FA are typically studied in white matter, where higher FA is generally interpreted as indicating greater directionality of water diffusion along myelinated tracts. ${ }^{34}$ The direction of the currently reported relationship suggests that higher FA correlates with greater basal ganglia injury, analogous to an earlier DTI study in CAA demonstrating increased FA in subthalamic gray matter relative to $\mathrm{HCs} .^{8}$ These data raise the possibility that when measured in subcortical gray matter nuclei, increased FA may indicate greater structural injury. Indeed, this idea has also been suggested in studies of striatum and pallidum in early Huntington's disease $\mathrm{e}^{35}$ and caudate in relapsing-remitting multiple sclerosis. ${ }^{36} \mathrm{~A}$ postulated mechanism for increased subcortical gray FA is targeted loss of dendritic connections causing restricted directional diffusion of the underlying fiber tracts. ${ }^{36}$

It remains to be determined whether CAA-related basal ganglia atrophy is a major contributor to the disease's clinical manifestations. Although we found relatively strong correlations between the extent of atrophy and two cognitive domains, only the association with language processing remained independent after controlling for covariates. This is noteworthy, especially considering how other types of CAA-related structural atrophy, such as white matter atrophy, affect different domains of cognitive function. ${ }^{7}$ It could thus be speculated that basal ganglia atrophy in patients with CAA is driven by a different underlying pathological mechanism and could contribute to the disease's long-term neurocognitive dysfunction. Previous studies in neurodegenerative diseases have indeed pointed to a possible role for basal ganglia atrophy in cognitive impairment. ${ }^{22,37}$ Given the potential for confounding by other CAA-related brain injuries, ${ }^{38}$ however, it remains unclear whether the correlation observed among the research CAA patients indicates direct functional impact of basal ganglia atrophy.

In addition to difficulty identifying the independent contribution of basal ganglia atrophy to CAA-related clinical dysfunction, the current analysis has other limitations. Even nondemented CAA patients typically have some degree of concomitant $A D$ pathology, raising the possibility that the identified basal ganglia atrophy is due to $A D$ rather than CAA. We found lower caudate, putamen, globus pallidus, and total BGVs in CAA relative to $A D$, however, arguing against this interpretation. Similarly, basal ganglia atrophy in D-CAA should be largely unconfounded by $A D$ pathology, as this condition has relatively few mature senile plaques and minimal neurofibrillary pathology. ${ }^{39}$ Another limitation is the use of controls and $A D$ scans drawn from ADNI for comparison to our CAA research and clinical cohorts, introducing the possibility of spurious differences due to variations in the MRI scans. We note that the ADNI patients were imaged using the same scanner type and magnetic field strength as the CAA group, and that the comparisons yielded basal ganglia regions that were both smaller in CAA than AD (caudate, putamen, globus pallidus) and smaller in $A D$ than $C A A$ (nucleus accumbens) suggesting no consistent inter-scanner bias. We also note that D-CAA patients and their matched controls were scanned on the same scanner and thus not affected by inter-scanner differences. Our analyses of HTN-ICH required a visual method for determining normalized basal ganglia width that could be applied to clinical-grade scans, potentially introducing inaccuracies in measurement relative to volumetric methods. The significant but not extremely strong correlation between the basal ganglia visual atrophy metric and the FreeSurfer-derived BGVs applied on the comparative $\mathrm{HC}$ cohort $(r=0.22)$ suggests that the visual atrophy scale can be used as a basal ganglia atrophy measure when volumetric measurement is not possible, but likely introduces measurement error into the comparison of the CAA-ICH and HTN-ICH patients. The measurement error, however, would be expected to bias towards a null result rather than towards a difference between the groups. The high inter-rater reliability of the visual scale suggests that the method is reproducible and can be tested in future cohorts. Finally, all our analyses were cross-sectional using MRls obtained at single timepoints. Longitudinal analysis of BGV will be an important future step for characterizing this process.

If confirmed in replication studies, the finding of basal ganglia atrophy would substantially extend the range of subcortical regions affected by $C A A$, raising challenging questions about the mechanisms for tissue injury distant from the amyloid-laden leptomeningeal and cortical vessels. Basal ganglia atrophy might also represent a novel neuroimaging marker of CAA-related brain injury. Previous studies have identified a range of candidate outcome markers for $C A A$, including both hemorrhagic and non-hemorrhagic brain lesions as well as alterations in vascular physiology. ${ }^{40}$ If future studies suggest that basal ganglia injury indeed contributes to the clinical manifestations characteristic of CAA, then this marker might play an important role in interventional trials aimed at limiting CAA-related neurologic dysfunction.

\section{Conclusions}

In this case-control study, patients with sporadic or hereditary CAA displayed significant atrophy in the basal ganglia com- 
pared to similar aged individuals without CAA. Moreover, CAA-related basal ganglia atrophy independently correlated with cortical gray matter atrophy, increased basal ganglia fractional anisotropy, and worse performance on language processing. Overall, the independent association of CAA with basal ganglia tissue loss could suggest a new mechanism for CAA-related brain injury and neurologic dysfunction, and a novel candidate biomarker of this currently untreatable disorder.

\section{Disclosure}

Jonathan Rosand reports personal fees from Boehringer Ingelheim, Pfizer, and New Beta Innovation. Steven M. Greenberg reports personal fees from Biogen, Hoffman-Laroche, and Washington University/IQVIA.

Jonathan Rosand reports funding from the National Institutes of Health (NIH) and OneMind. M. Edip Gurol reports research funding from NIH, Avid Radiopharmaceuticals (wholly owned subsidiary of Eli Lilly), Boston Scientific, and Pfizer. Steven M. Greenberg reports funding from NIH.

\section{Acknowledgments}

Part of the data collection and sharing for this project was funded by the Alzheimer's Disease Neuroimaging Initiative (ADNI) (National Institutes of Health Grant U01 AG024904) and DOD ADNI (Department of Defense award number W81XWH-12-2-0012). ADNI is funded by the National Institute on Aging, the National Institute of Biomedical Imaging and Bioengineering, and through generous contributions from the following: AbbVie, Alzheimer's Association, Alzheimer's Drug Discovery Foundation, Araclon Biotech, BioClinica Inc., Biogen, Bristol-Myers Squibb Company, CereSpir Inc., Cogstate, Eisai Inc., Elan Pharmaceuticals Inc., Eli Lilly and Company, Eurolmmun, and F. Hoffmann-La Roche Ltd.; and its affiliated company Genentech Inc., Fujirebio, GE Healthcare, IXICO Ltd., Janssen Alzheimer Immunotherapy Research \& Development LLC., Johnson \& Johnson Pharmaceutical Research \& Development LLC., Lumosity, Lundbeck, Merck \& Co. Inc., Meso Scale Diagnostics LLC., NeuroRx Research, Neurotrack Technologies, Novartis Pharmaceuticals Corporation, Pfizer Inc., Piramal Imaging; Servier, Takeda Pharmaceutical Company, and Transition Therapeutics. The Canadian Institutes of Health Research is providing funds to support ADNI clinical sites in Canada. Private sector contributions are facilitated by the Foundation for the National Institutes of Health (www.fnih.org). The grantee organization is the Northern California Institute for Research and Education, and the study is coordinated by the Alzheimer's Therapeutic Research Institute at the University of Southern California. ADNI data are disseminated by the Laboratory for Neuro Imaging at the University of Southern California.

\section{References}

1. Wardlaw JM, Smith EE, Biessels GJ, Cordonnier C, Fazekas F, Frayne $R$, et al. Neuroimaging standards for research into small vessel disease and its contribution to ageing and neurodegeneration. Lancet Neurol 2013;12:822-838.

2. Nitkunan $A$, Lanfranconi $S$, Charlton RA, Barrick TR, Markus HS. Brain atrophy and cerebral small vessel disease: a prospective follow-up study. Stroke 2011;42:133-138.

3. Ikram MK, de Jong FJ, Vernooij MW, Hofman A, Niessen WJ, van der Lugt $A$, et al. Retinal vascular calibers associate differentially with cerebral gray matter and white matter atrophy. Alzheimer Dis Assoc Disord 2013;27:351-355.

4. Brundel $M$, van den Heuvel $M$, de Bresser J, Kappelle $\sqcup$, Biessels GJ; Utrecht Diabetic Encephalopathy Study Group. Cerebral cortical thickness in patients with type 2 diabetes. J Neurol Sci 2010;299:126-130.

5. Fotiadis , van Rooden $S$, van der Grond J, Schultz A, Martinez-Ramirez $S$, Auriel $E$, et al. Cortical atrophy in patients with cerebral amyloid angiopathy: a case-control study. Lancet Neurol 2016;15:811-819.

6. Gurol ME, Viswanathan A, Gidicsin C, Hedden T, Martinez-Ramirez $S$, Dumas $A$, et al. Cerebral amyloid angiopathy burden associated with leukoaraiosis: a positron emission tomography/magnetic resonance imaging study. Ann Neurol 2013;73:529-536.

7. Fotiadis $P$, Reijmer YD, Van Veluw SJ, Martinez-Ramirez $S$,

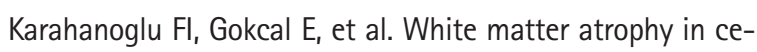
rebral amyloid angiopathy. Neurology 2020;95:e554-e562.

8. Salat DH, Smith EE, Tuch DS, Benner T, Pappu V, Schwab KM, et al. White matter alterations in cerebral amyloid angiopathy measured by diffusion tensor imaging. Stroke 2006;37: 1759-1764.

9. Reijmer YD, Fotiadis $P$, Martinez-Ramirez $S$, Salat DH, Schultz A, Shoamanesh A, et al. Structural network alterations and neurological dysfunction in cerebral amyloid angiopathy. Brain 2015;138(Pt 1):179-188.

10. Soontornniyomkij V, Lynch MD, Mermash S, Pomakian J,

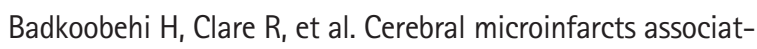
ed with severe cerebral beta-amyloid angiopathy. Brain Pathol 2010;20:459-467.

11. Auriel $E$, Edlow BL, Reijmer YD, Fotiadis $P$, Ramirez-Martinez $\mathrm{S}, \mathrm{Ni}$ J, et al. Microinfarct disruption of white matter structure: 
a longitudinal diffusion tensor analysis. Neurology 2014; 83:182-188.

12. Dumas A, Dierksen GA, Gurol ME, Halpin A, MartinezRamirez $S$, Schwab $K$, et al. Functional magnetic resonance imaging detection of vascular reactivity in cerebral amyloid angiopathy. Ann Neurol 2012;72:76-81.

13. Peca S, McCreary CR, Donaldson E, Kumarpillai G, Shobha N, Sanchez $\mathrm{K}$, et al. Neurovascular decoupling is associated with severity of cerebral amyloid angiopathy. Neurology 2013;81:1659-1665.

14. Mink JW. The basal ganglia and involuntary movements: impaired inhibition of competing motor patterns. Arch Neurol 2003;60:1365-1368.

15. Foerde K, Shohamy D. The role of the basal ganglia in learning and memory: insight from Parkinson's disease. Neurobiol Learn Mem 2011;96:624-636.

16. Booth JR, Wood L, Lu D, Houk JC, Bitan T. The role of the basal ganglia and cerebellum in language processing. Brain Res 2007;1133:136-144.

17. Tanaka SC, Doya K, Okada G, Ueda K, Okamoto Y, Yamawaki $S$. Prediction of immediate and future rewards differentially recruits cortico-basal ganglia loops. Nat Neurosci 2004;7: 887-893.

18. Ashar YK, Andrews-Hanna JR, Dimidjian S, Wager TD. Empathic care and distress: predictive brain markers and dissociable brain systems. Neuron 2017;94:1263-1273.

19. Tinaz S, Courtney MG, Stern CE. Focal cortical and subcortical atrophy in early Parkinson's disease. Mov Disord 2011;26: 436-441.

20. Cordato NJ, Halliday GM, Harding AJ, Hely MA, Morris JG. Regional brain atrophy in progressive supranuclear palsy and Lewy body disease. Ann Neurol 2000;47:718-728.

21. Aylward EH, Li $Q$, Stine OC, Ranen $N$, Sherr M, Barta PE, et al. Longitudinal change in basal ganglia volume in patients with Huntington's disease. Neurology 1997;48:394-399.

22. Hanganu A, Provost JS, Monchi O. Neuroimaging studies of striatum in cognition part II: Parkinson's disease. Front Syst Neurosci 2015;9:138.

23. Greenberg SM, Charidimou A. Diagnosis of cerebral amyloid angiopathy: evolution of the Boston criteria. Stroke 2018;49: 491-497.

24. Jack CR Jr, Bernstein MA, Fox NC, Thompson P, Alexander G, Harvey $D$, et al. The Alzheimer's Disease Neuroimaging Initiative (ADNI): MRI methods. J Magn Reson Imaging 2008;27: 685-691.

25. van Rooden S, van der Grond J, van den Boom R, Haan J, Linn J, Greenberg SM, et al. Descriptive analysis of the Boston criteria applied to a Dutch-type cerebral amyloid angiopathy population. Stroke 2009;40:3022-3027.

26. Pasi M, Boulouis G, Fotiadis P, Auriel E, Charidimou A, Haley $K_{1}$ et al. Distribution of lacunes in cerebral amyloid angiopathy and hypertensive small vessel disease. Neurology 2017; 88:2162-2168.

27. Fischl B, Dale AM. Measuring the thickness of the human cerebral cortex from magnetic resonance images. Proc Natl Acad Sci U S A 2000;97:11050-11055.

28. Lee MJ, Kim TH, Kim SJ, Kim BK, Mun CW, Lee JH. Quantitative validation of a visual rating scale for defining high-iron putamen in patients with multiple system atrophy. Front Neurol 2019;10:1014.

29. Case NF, Charlton A, Zwiers A, Batool S, McCreary CR, Hogan $\mathrm{DB}$, et al. Cerebral amyloid angiopathy is associated with executive dysfunction and mild cognitive impairment. Stroke 2016;47:2010-2016.

30. Selden N, Mesulam MM, Geula C. Human striatum: the distribution of neurofibrillary tangles in Alzheimer's disease. Brain Res 1994;648:327-331.

31. Gray F, Dubas F, Roullet E, Escourolle R. Leukoencephalopathy in diffuse hemorrhagic cerebral amyloid angiopathy. Ann Neurol 1985;18:54-59.

32. Smith EE, Nandigam KR, Chen YW, Jeng J, Salat D, Halpin $A$, et al. MRI markers of small vessel disease in lobar and deep hemispheric intracerebral hemorrhage. Stroke 2010;41:19331938.

33. Fisher CM. Pathological observations in hypertensive cerebral hemorrhage. J Neuropathol Exp Neurol 1971;30:536-550.

34. Werring DJ, Clark CA, Barker GJ, Thompson AJ, Miller DH. Diffusion tensor imaging of lesions and normal-appearing white matter in multiple sclerosis. Neurology 1999;52:16261632.

35. Douaud G, Behrens TE, Poupon C, Cointepas Y, Jbabdi S,

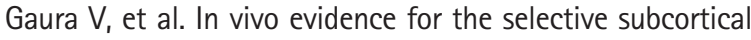
degeneration in Huntington's disease. Neuroimage 2009;46: 958-966.

36. Hasan KM, Halphen C, Kamali A, Nelson FM, Wolinsky JS, Narayana PA. Caudate nuclei volume, diffusion tensor metrics, and $T(2)$ relaxation in healthy adults and relapsing-remitting multiple sclerosis patients: implications for understanding gray matter degeneration. J Magn Reson Imaging 2009;29:70-77.

37. Aylward EH, Harrington DL, Mills JA, Nopoulos PC, Ross CA, Long JD, et al. Regional atrophy associated with cognitive and motor function in prodromal Huntington disease. J Huntingtons Dis 2013;2:477-489.

38. Gurol ME, Irizarry MC, Smith EE, Raju S, Diaz-Arrastia R, Bottiglieri $T$, et al. Plasma beta-amyloid and white matter le- 
sions in $\mathrm{AD}, \mathrm{MCl}$, and cerebral amyloid angiopathy. Neurology 2006;66:23-29.

39. Maat-Schieman ML, Radder CM, van Duinen SG, Haan J, Roos RA. Hereditary cerebral hemorrhage with amyloidosis (Dutch): a model for congophilic plaque formation without neurofibrillary pathology. Acta Neuropathol 1994;88:371-
378.

40. Greenberg SM, Al-Shahi Salman R, Biessels GJ, van Buchem M, Cordonnier C, Lee JM, et al. Outcome markers for clinical trials in cerebral amyloid angiopathy. Lancet Neurol 2014;13: 419-428. 